Using information technology to improve linkage into sexual health care for patients receiving HIV post exposure prophylaxis for sexual exposure (PEPSE) in

\section{Emergency Departments}

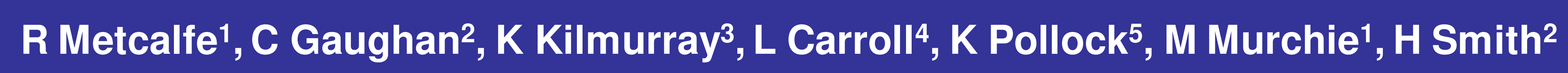

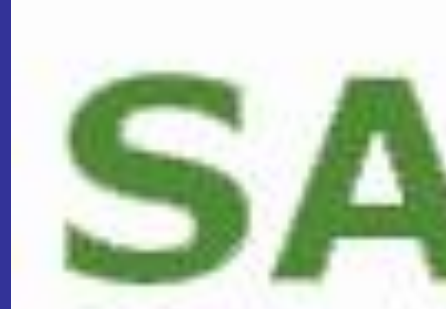

\title{
Background
}

HIV PEPSE should be commenced within 72 hours (ideally 24 hours) after possible exposure to HIV.

Patient education on PEPSE includes advice on attendance at Emergency Department (ED) if outside opening hours of local sexual health services.

Our healthboard serves a population of 1.1 million with four EDs. An initial four month audit revealed 12 patients received a 5-day starter pack of PEPSE at ED and there was no communication between departments; patients were told to selfrefer to sexual health services.

HIV testing at baseline was also poor in this setting (75\%), highlighting importance of linkage into sexual health services after presentation of possible HIV exposure, in this high risk group.

We recognised there was no robust mechanism to ensure these high risk patients were not lost to follow up (LTFU) after receiving HIV PEPSE in EDs and HIV testing rates in EDs for this presentation was low.

Aim

To provide a robust mechanism of referral to sexual health services after presentation to EDs for HIV PEPSE

Interventions

HIV PEPSE 5 day starter pack leaflets were altered to ask the dispensing clinician to refer patient via secure email or telephone message to the sexual health advisers at sexual health service.
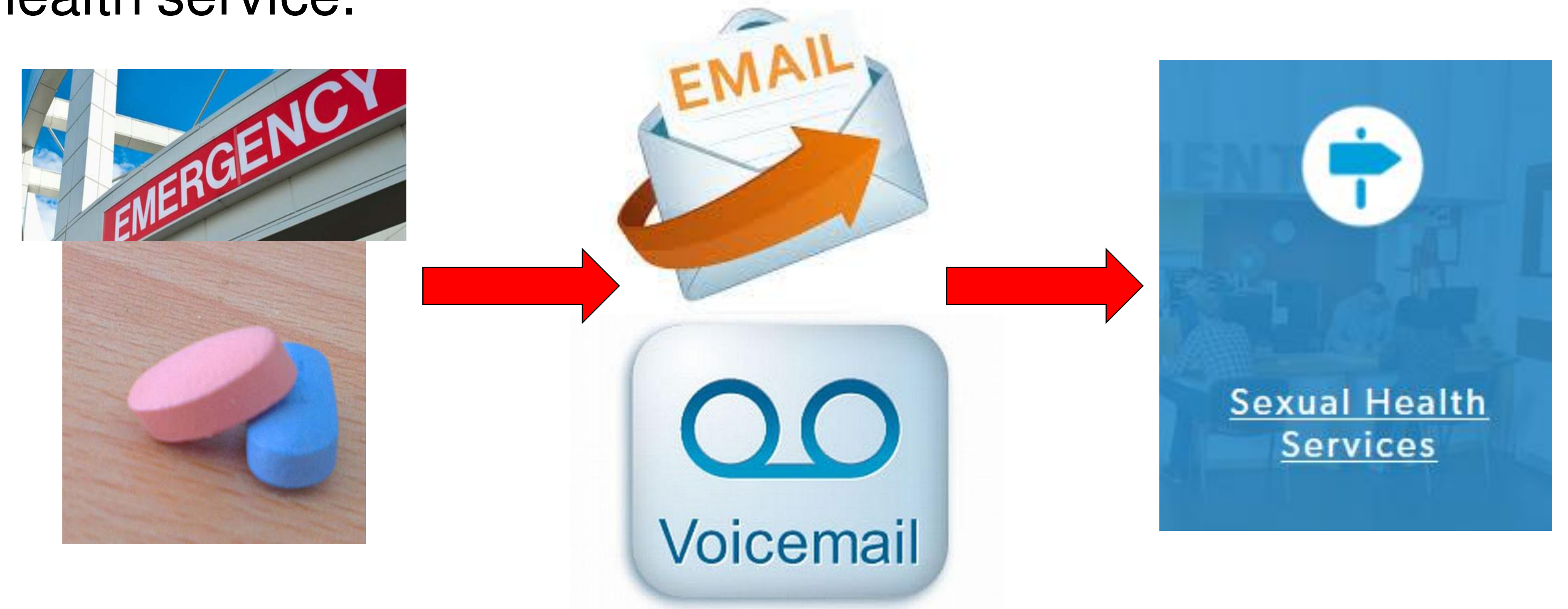

Interventions

-ED physicians in 2 main EDs championed the new referral system to encourage colleagues

- Teaching on HIV testing and PEPSE was delivered and ED physicians encouraged HIV testing amongst staff
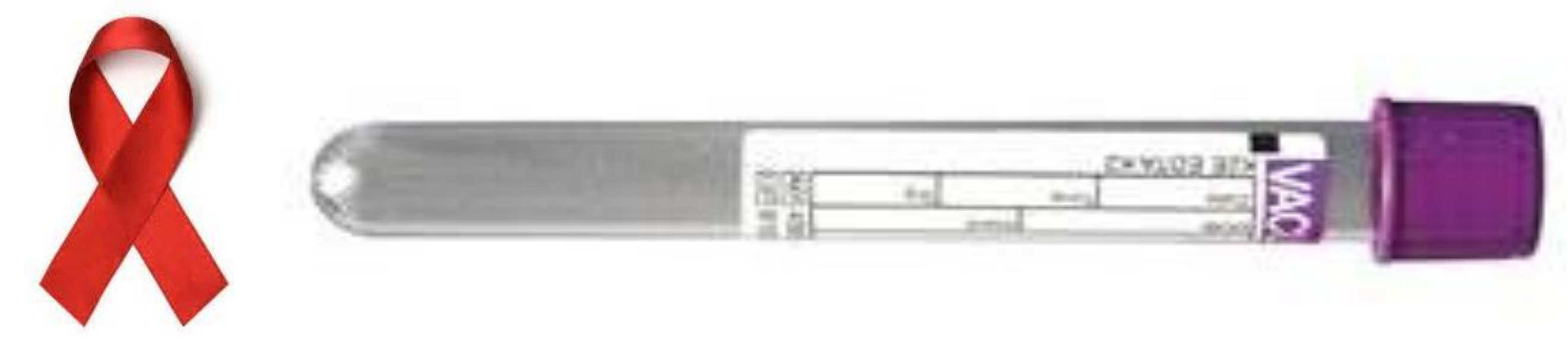

-HIV PEPSE prescriptions and referrals to SHS was prospectively audited for a 4 month period and monthly results fed back

Results

Prospective four month re-audit showed an increase in HIV PEPSE prescriptions from Emergency Departments to 19. $12 / 19$ (63\%) were referred by email and 6/19 (32\%) via answering machine message; $1 / 19$ (5\%) self referred only. No patient found this referral unacceptable; all subsequently attended sexual health services for continuation pack (100\%). All 19 (100\%) were male, aged between 18 and 46. 17/19 (89\%) were MSM.

$11 / 19(60 \%)$ had reported condomless receptive anal intercourse. The remaining $40 \%$ reported condomless insertive anal intercourse or condomless insertive vaginal intercourse

16/19 (84\%) completed 28 days HIV PEPSE; 1/19 DNA any further follow up and 2/19 stopped the medication.

$17 / 19(89 \%)$ have had 8 week post HIV PEPSE completion HIV testing and all 17 were negative.

$5 / 19(26 \%)$ had a baseline HIV test performed; of those that didn't, all had an HIV test performed at first presentation to sexual health services.

\section{Conclusions}

Following implementation of the email/telephone referral intervention, we found an increased number of patients received HIV PEPSE from EDs in the health board area and all were successfully linked into sexual health services. We cannot be sure that this increase is due to the prevention of patients being lost to follow up between the two services; other reasons include an increased awareness of PEPSE and where to obtain this out of hours.

We found poor baseline HIV testing rates in EDs, despite specific training and in house HIV testing champions. Therefore, this robust referral system to sexual health services is even more crucial for this group of people who are at high risk of HIV infection and for whom, HIV testing is a priority. 